\title{
Erector spinae muscle responses while standing
}

\author{
NEZIHE ERTEKIN AND CUMHUR ERTEKIN \\ From the Departments of Physical Therapy and Rehabilitation and Neurology, Aegean University \\ Medical School Hospital, Bornova-Izmir, Turkey.
}

S U M MARY In 55 normal adult subjects standing erect, stable EMG responses could be recorded from the lumbar erector spinae muscles in response to unilateral sudden arm abduction produced by single electrical stimuli applied to the brachial plexus at Erb's point, or to knee and ankle movement produced by stimuli to the posterior tibial nerve in the popliteal fossa.

The influence of gravitational and external forces on standing posture and the compensatory mechanisms to these forces can be observed clinically by body sway and recorded either by platform studies, ${ }^{1-5}$ or by other electrophysiological methods. ${ }^{6}$ In this paper a new and simple method of recording the compensatory responses to gravitational forces while standing is described. Regular electromyographic (EMG) responses can be obtained from the lumbar erector spinae muscles in response to unilateral sudden arm abduction, or to knee flexion and ankle extension, produced by single electrical stimuli applied to the brachial plexus at the Erb's point, or to the posterior tibial nerve in the popliteal fossa respectively.

\section{Method}

Fifty-five normal control subjects were investigated (14 female and 41 male, mean age $37 \cdot 9$ years, range 14 to 74 years). The majority of the subjects were in the age group 20-50 years.

A simple diagram of the electrophysiological method is illustrated in fig 1 . Subjects were asked to stand with the feet about $20 \mathrm{~cm}$ apart on a hard and stable wooden platform. Teflon-coated stainless steel needle electrodes were inserted into the lumbar erector spinae muscles bilaterally at the 2 nd or 3 rd vertebral process and about $1.5 \mathrm{~cm}$ lateral to the midline. A reference electrode was inserted subcutaneously in the midline just above the vertebral process. In some cases a third needle electrode was inserted into the paravertebral muscles located around the mid-thoracic vertebral level. The active electrode had a bare tip of $3 \mathrm{~mm}$ and the reference electrode had a bare tip of $4 \mathrm{~mm}$ (DISA $9013 \mathrm{~K}$

Address for reprint requests: Prof C Ertekin, Dept of Neurology, Medical School Hospital, Bornova-Izmir, Turkey.

Accepted 30 July 1980
5141). The recording electrodes were connected to a standard EMG amplifier (DISA 14 A 30).

The lumbar erector spinae muscle EMG activity varied in intensity while standing, but complete electrical silence could be obtained by postural adjustments such as by increasing the lumbar lordosis slightly or by backward movement of the shoulders. These postural adjustments were carried out by the subjects, of ten with the examiner's help. When the paravertebral muscles were electrically silent, the brachial plexus at Erb's point or the posterior tibial nerve in the popliteal fossa were stimulated randomly via surface bipolar stimulating electrodes (DISA $13 \mathrm{~K}$ 62) connected to the output of a stimulator (DISA Ministim 14 E 10), with single rectangular electrical pulses of $0.2 \mathrm{~ms}$ duration and $300-500 \mathrm{~V}$ in strength.

\section{Results}

In all normal subjects, stimulation of the brachial plexus at Erb's point or of the posterior tibial nerve in the popliteal fossa, evoked EMG responses in the lumbar erector spinae muscles

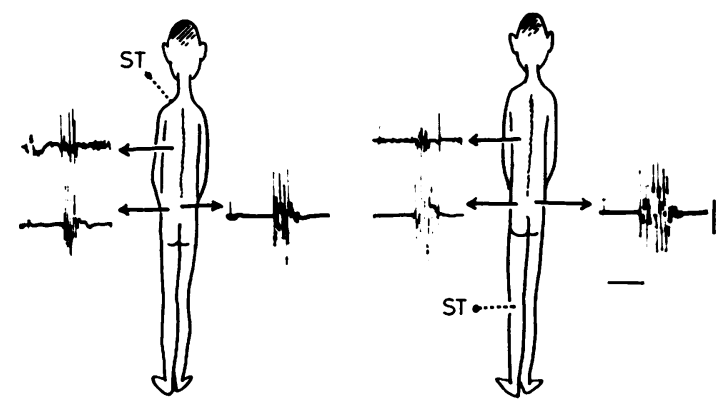

Fig 1 Thoracic and lumbar erector spinae muscle response obtained by single stimulation (ST) of the left brachial plexus at Erb's point and the left posterior tibial nerve in the popliteal fossa. Calibration: $0 \cdot 2 \mathrm{mV}$ and $100 \mathrm{~ms}$. 

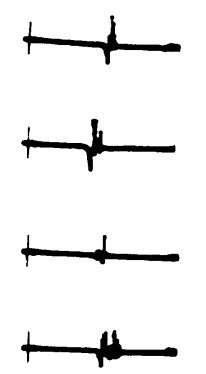

Fig 2 Electromyographic recordings showing fluctuation in amplitude and latency of the erector spinae muscle responses obtained by single stimulation at random intervals of the brachial plexus at Erb's point. Calibration: $0.2 \mathrm{mV}$ and $100 \mathrm{~ms}$.

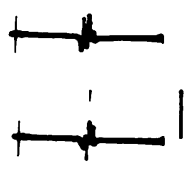

bilaterally with each electrical stimulus (fig 1). These muscle responses fluctuated in latency, shape and amplitude with each stimulus (fig 2). The responses appeared quite stable so that an average latency could be calculated. Peak to peak amplitude of the highest response was taken in each series of recording conditions (table). As long as the subjects maintained their position, the responses almost always were obtained for each stimulus; sometimes another response of longer latency could be observed, but this late response tended to fluctuate and to disappear from one stimulus to another (fig 3).

The latency of the lumbar erector spinae muscle responses obtained by stimulation at Erb's point was shorter than that of those obtained by stimulation in the popliteal fossa (fig 4). The average latency to the onset of the first response was about $88 \mathrm{~ms}$ for Erb's point stimulation (75 observations), while it was about $105 \mathrm{~ms}$ for popliteal fossa stimulation (60 observations)
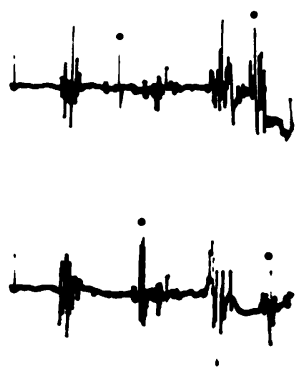

Fig 3 The early and the late unstable (black circles) erector spinae muscle EMG responses evoked by single stimulation at Erb's point (2 stimuli are shown on each trace). Calibration: $0 \cdot 2 \mathrm{mV}$ and $100 \mathrm{~ms}$.

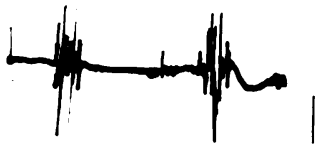

$(\mathrm{p}<0.001)$. On the other hand, late and nonstable responses appeared earlier with popliteal fossa stimulation than with Erb's point stimulation. Their latencies were about $205-207 \mathrm{~ms}$ with popliteal fossa stimulation compared to 214$221 \mathrm{~ms}$ with Erb's point stimulation. The inci-

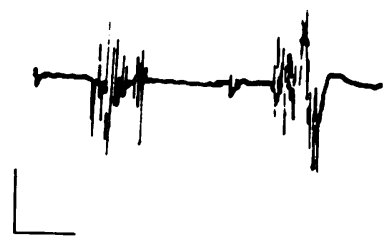

Fig 4 Erector spinae muscle responses from both sides obtained by single stimulation of right Erb's point (upper traces) and left popliteal fossa (lower traces). The latency of the EMG responses is longer in

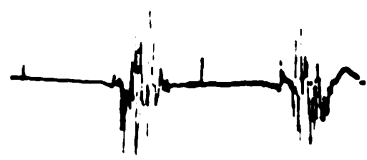
the stimulation of popliteal fossa. Calibration: $0.2 \mathrm{mV}$ and $100 \mathrm{~ms}$.

Table Comparison of the erector spinae muscle responses obtained by stimulation either of the brachial plexus at Erb's point, or of the posterior tibial nerve in the popliteal fossa (Means $\pm 1 S E M$, and 1 SD, with the range in brackets)

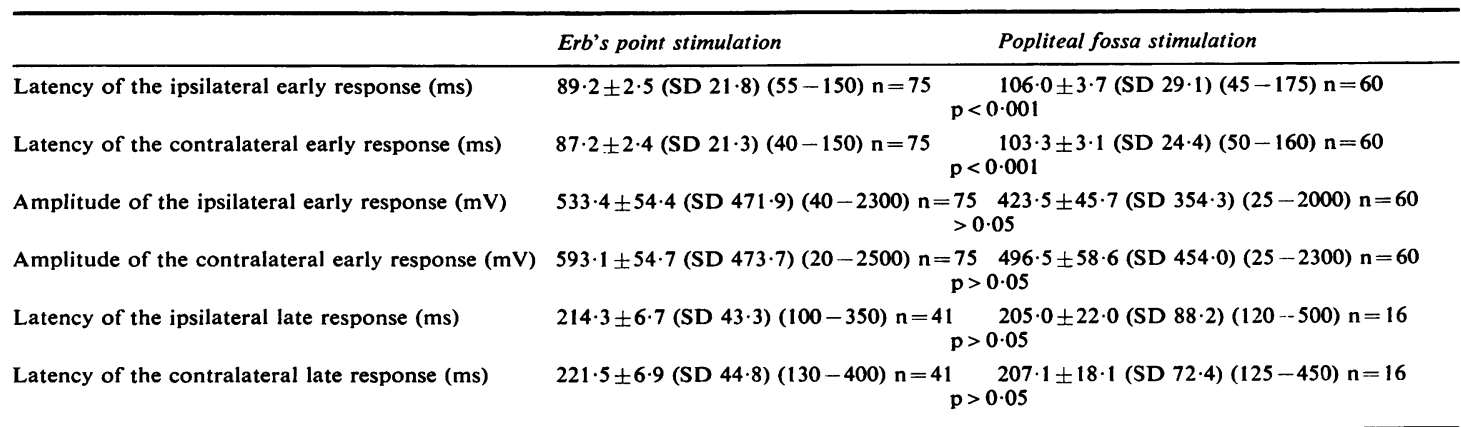



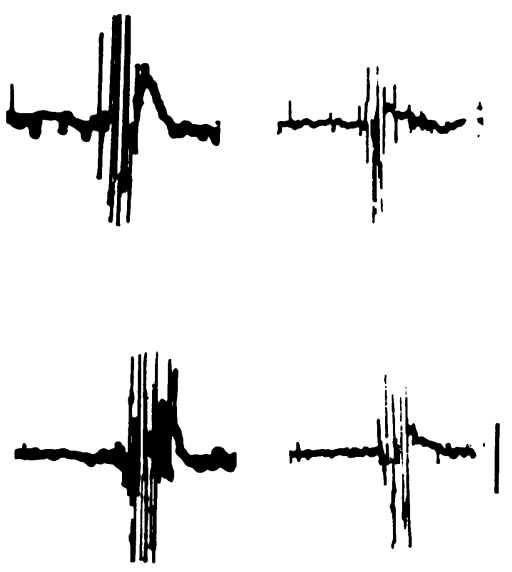

Fig 5 Erector spinae muscle responses obtained by single stimulation of left Erb's point (upper traces) and left popliteal fossa (lower traces). Contralateral EMG responses (on the left column) are denser and larger.

Calibration: $0 \cdot 1 \mathrm{mV}$ and $100 \mathrm{~ms}$.

dence of late responses recorded from both sides was $68 \%$ with stimulation ipsilateral to Erb's point and $59 \%$ with stimulation contralateral to Erb's point; the incidence of late responses was lower with popliteal fossa stimulation, being $33 \%$ and $30 \%$ respectively for all the 55 normal subjects. Late responses obtained from only single side of recordings were not included into the calculations. The erector spinae muscle responses to stimulation in the contralateral popliteal fossa or at Erb's point were somewhat larger in size. But this observation was not statistically significant (fig 5). As mentioned above, when complete electrical silence was obtained in the lumbar
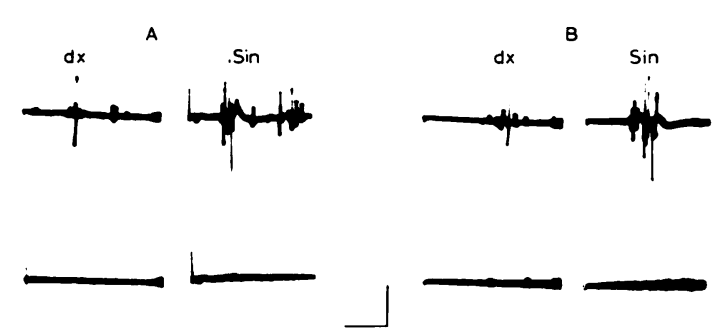

Fig 6 Upper traces: Right $(d x)$ and left (sin) erector spinae muscle responses obtained by single stimulation of right Erb's point (A) and right popliteal fossa (B) while standing at ease. Lower traces: The same stimulation and recording conditions, but the lumbar lordosis was increased. Note the loss of responses. Calibration: $0.5 \mathrm{mV}$ and $100 \mathrm{~ms}$. erector spinac muscles by postural adjustments such as slight increase of lumbar lordosis or backward movement of the shoulders, electrical stimuli applied to the Erb's point or popliteal fossa produced EMG responses in these muscles; but a further increase of lumbar lordosis greatly reduced or abolished the evoked EMG responses (fig 6).

When four subjects were lying prone, no responses were evoked at the lumbar erector spinae muscles by stimulating either the brachial plexus or the posterior tibial nerve with the same stimulation and recording conditions (fig 7).

The responsiveness of the lumbar erector spinae muscles to stimulation of other nerves also was investigated in 24 normal subjects while standing. There were no responses to a supramaximal stimulation of the median or ulnar nerves at the level of the elbow, of the peroneal nerve at the head of the fibula, of the sural nerve at the lateral malleolus, and even to stimulation of the posterior tibial nerve at the medial malleolus. The effect of strong skin stimulation on the distal or proximal part of the skin was not studied extensively, but painful electrical stimuli applied to the sural nerve at the lateral malleolus did not evoke consistent responses from the erector spinae muscles.

The effects of electrical stimuli could not easily be imitated by sudden voluntary abduction of one arm, or by sudden voluntary flexion at the knee; the resulting contralateral EMG activity was often earlier in onset and longer in duration.

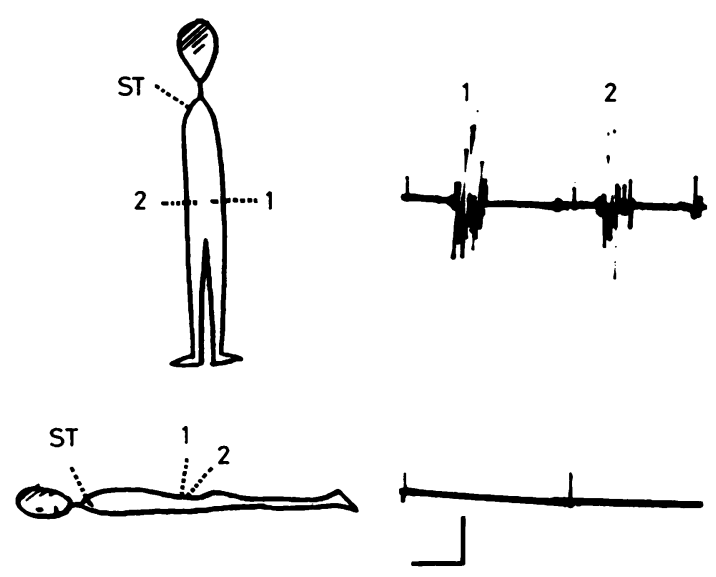

Fig 7 Upper traces: right (1) and left (2) erector spinae muscle responses obtained by single stimulation at left Erb's point while standing at ease. Lower traces: The same stimulation and recording conditions, but lying prone. Note the loss of responses. Calibration: $0.5 \mathrm{mV}$ and $100 \mathrm{~ms}$. 
However, on several attempts at brisk voluntary arm abduction, quite variable EMG discharges were obtained from the lumbar erector spinae muscles. Latency value of contralateral erector spinae muscles often varied between $40-170 \mathrm{~ms}$ after the onset of arm abduction while the response from the ipsilateral erector spinae muscles appeared about $60-200 \mathrm{~ms}$ after arm abduction. The latency values were often $40-110 \mathrm{~ms}$ shorter in contralateral erector muscles for each voluntary attempt (fig 8). Sudden abduction of the arm from the shoulder seems to be critical for the lumbar erector spinae muscle response. If both arms were fixed to the trunk by a bandage the response to electrical stimulation at Erb's point was considerably diminished but not abolished (fig 9).

The behaviour of some other muscles also was investigated in a few subjects during stimulation of Erb's point or popliteal fossa or both. The soleus muscle did not respond to stimulation to
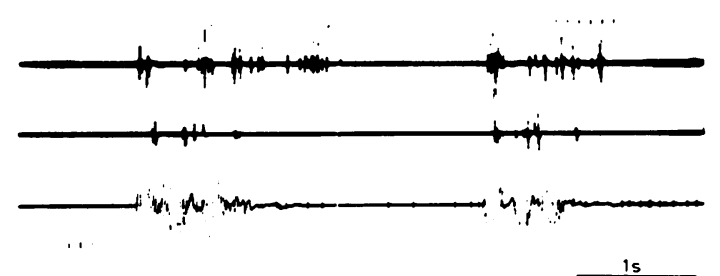

Fig 8 Continuous recording from left (upper trace) and right (middle trace) erector spinae and right deltoid (lower trace) muscles. Abduction of the right arm causes EMG discharges of the erector spinae muscles with earlier onset in the contralateral side.

A

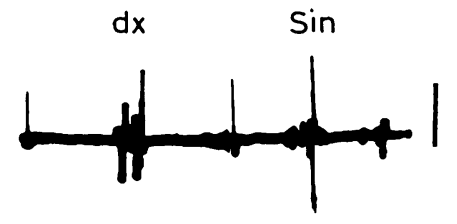

B

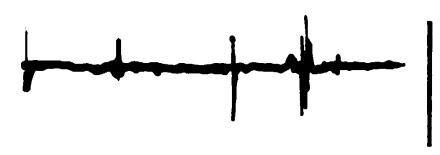

Fig 9 Right $(d x)$ and left (sin) erector spinae muscle responses obtained by single stimulation of left Erb's point in easy standing posture (A) and after fixation of the arms to the trunk (B). Note the reduction of the responses. Calibration: $0 \cdot 2 \mathrm{mV}$ and $200 \mathrm{~ms}$.
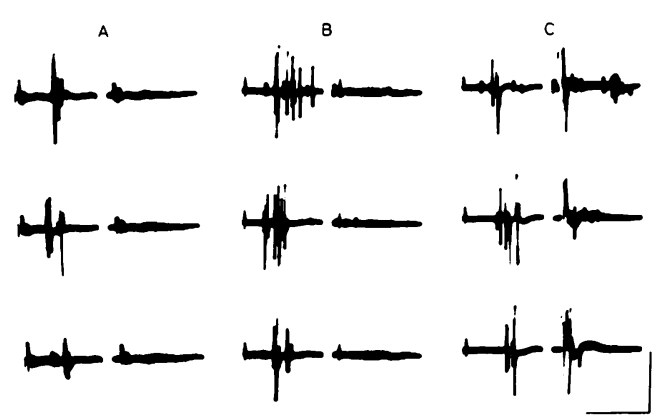

Fig 10 The EMG recordings from the left erector spinae muscle (on the left columns) and right soleus muscle (on the right columns) obtained by single stimulation of the right Erb's point (A), left (B) and right (C) popliteal fossa. Note that only ipsilateral popliteal fossa stimulation causes an EMG response other than direct $M$ response in soleus muscle. Calibration: $0 \cdot 2 \mathrm{mV}$ and $200 \mathrm{~ms}$.

Erb's point or contralateral popliteal fossa at least for $250 \mathrm{~ms}$ analysis time; only very labile late responses other than direct M-response could be seen during ipsilateral popliteal fossa stimulation (fig 10).

In a normal subject, both Erb's point and popliteal fossa stimulation evoked EMG responses in the rectus abdominis muscle similar to those obtained in erector spinae muscles. Furthermore both Erb's point and popliteal fossa stimulation also could produce similar responses in the thoracic erector spinae muscles, but they were more labile and sometimes were absent (fig 1).

\section{Discussion}

Applying single supramaximal electrical stimuli of a strength below the pain threshold to the brachial plexus at Erb's point or to the posterior tibial nerve at the popliteal fossa, produced EMG responses in the lumbar erector spinae muscles in the relaxed quietly standing normal subject. No such muscle responses were obtained when lying. This suggests that the lumbar erector spinae muscle responses are directly related to antigravity mechanisms. A very rapid and large abduction movement of the arm at the shoulder joint occurred upon stimulation of the brachial plexus at Erb's point. This movement causes a suddent shift of the centre of gravity laterally, so a fast compensatory adjustment was needed. This can be achieved by the contraction of lumbar erector spinae muscles within $100 \mathrm{~ms}$. Sudden plantar flexion around the ankle joint and mild knee flexion was produced by stimu- 
lation of the posterior tibial nerve; causing a shift of the centre of gravity in both lateral and antero-posterior directions because of the sudden rise of the heel from the ground. Since the trunk consists of a spring lattice with distributed mass, each limb upthrust must be expected to produce some distortion of the trunk. ${ }^{7}$ The trunk muscles, including erector spinae muscles are crucially important in adjusting to sudden changes of the gravity line due to strong limb movements. More distal phasic movements produced by the stimulation of the peripheral nerves at points distal to the shoulder or knee joints did not cause any EMG responses in the lumbar erector spinae muscles, perhaps because they did not change the centre of gravity. The voluntary imitation of electrically induced phasic movements of the arm and leg resulted in similar EMG changes in the lumbar erector spinae muscles, an observation described in kinesiological EMG studies in which abduction of the arm also produced contractions in the lumbar paraspinal muscles. 910

Although the erector spinae muscle responses appeared bilaterally upon unilateral stimulation, the contralateral EMG responses were generally greater than those recorded ipsilaterally. This finding indicates that the shift of the centre of gravity in the lateral direction could be corrected by stronger contralateral compensatory contractions in order to keep balance. Lumbar erector spinae muscle responses obtained only during erect posture could be different from the other generalised muscle reactions such as microreflexes, ${ }^{11-13}$ startle and orienting reactions ${ }^{14-16}$ and even voluntary reactions. Microreflexes, startle and orienting reactions can be excluded for the following reasons: (1) They are easily evoked by different kind of sensory stimuli, for example, visual, auditory and somatic stimuli. (2) They are often obtained in the recumbent position and their relation to the erect posture has not been demonstrated. (3) They are usually very small in size and there may be a need for computer averaging of muscle responses. (4) Their latencies are increased as more caudal muscles investigated. A voluntary reaction to the electrical stimuli also is unlikely to be responsible; the average voluntary reaction time should not be faster than $140 \mathrm{~ms},{ }^{17-18}$ but the first stable erector spinae muscle responses obtained in this study was much earlier

Figure 11 shows a diagram of the hypothetical pathways and circuits of the early erector spinae muscle responses evoked by stimulation of Erb's point or in the popliteal fossa. It is suggested

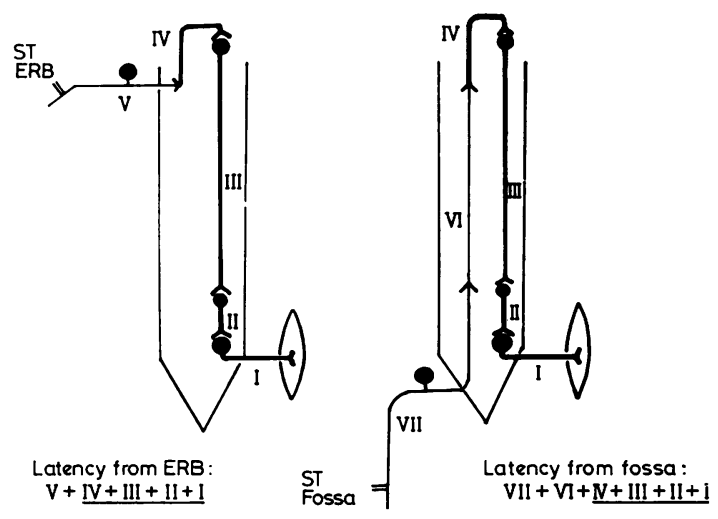

Fig 11 A diagram showing hypothetical pathways of the early erector spinae muscle responses evoked by stimulation of Erb's point or in the popliteal fossa.

that the dorsal columns may be involved in the afferent route of such a long loop antigravitational mechanism. The efferent routes (denoted as I, II, III) and some probable ascending supraspinal pathways (denoted as IV) and relays in this circuit are the same for both stimulation sites; but the remaining afferent pathways involved in the system obviously could be different. Thus the afferent volley from the leg is carried throughout from peripheral afferent fibres to the lumbosacral spinal cord (denoted as VII) and from this region to the cervical cord (denoted as VI), while the impulses from peripheral nerve fibres reach the cervical cord (denoted as V) in the case of the arm. The latency of EMG response on popliteal fossa stimulation was found to be about $15-20$ ms longer than that on stimulation of Erb's point. This time difference is close to the value of "cervical tractus response" recorded intrathecally at the cervical cord level in man by stimulation of the posterior tibial nerve in the popliteal fossa. ${ }^{19}$ The time taken from stimulation of Erb's point to the cord (denoted as V) must be very small, about 1-2 ms, because the latency to most of the cervical segmental cord evoked potential recorded intrathecally is about $6 \mathrm{~ms}$ on stimulation of the median nerve at the elbow. ${ }^{19}$ Therefore, the approximate value of $15-20 \mathrm{~ms}$ latency difference suggests that the afferent impulses for such responses are conveyed through the dorsal columns in which the impulses are carried very quickly. Furthermore the tractus response has been shown to originate mainly from activation of the dorsal column fibres. ${ }^{19-22}$ The mechanism and the neural pathways of such antigravitational responses needs further studies. One of the 
approaches to solve this is to apply the method on patients with various central nervous system disorders, and such a study is under investigation in our laboratory.

\section{References}

1 Begbie GH. Some problems of postural sway. In: de Reuck AVS, Julie Knight, eds. Myotatic Kinesthetic and vestibular mechanisms. Ciba Foundation Symposium. London: J A Churchill Ltd, 1967: 80-92.

2 Begbie GH. The assessment of imbalance. Physiotherapy 1969; 55:411-4.

3 Nasher LM. Vestibular and reflex control of normal standing. In: Stein RB, Pearson KG, Smith RS, Redford JB, eds. Control of posture and locomotion. New York, London: Plenum Press, 1973: 291-308.

4 Gantchev G, Dunev S, Draganova N. On the spontaneous and induced body oscillations. In: Gydikov AA, Tantov NT, Kosarov DS, eds. Motor control. New York, London: Plenum Press, 1973: 179-94.

5 Gurfinkel VS, Lipshits MI, Mori S, Popov KE. The state of stretch reflex during quiet standing in man. In: Homma S, ed. Progress in Brain Research, vol 44. Amsterdam: Elsevier, 1976: 473-86.

6 Floyd WF, Silver PHS. The function of the erectores spinae muscles in certain movements and postures in man. J Physiol 1955; 129:184203.

7 Roberts TDM. The mechanics of upright posture. Physiotherapy 1969; 55:398-404.

8 Roberts TDM. The role of vestibular and neck receptors in locomotion. In: Herman RM, Grillner S, Stein PSG, Stuart DG, eds. Advances in Behavioural Biology, vol 18. Neural control of locomotion. New York: Plenum Press, 1976: 539-60.

9 Portnoy H, Morin F. Electromyographic study of postural muscles in various positions and movements. Am J Physiol 1956; 186:122-6.

10 Hufschmidt HJ. Questions on tremor and ataxia.
Electroencephalogr Clin Neurophysiol suppl 1962; 22:114-9.

11 Bickford RG. Fast motor systems in man. Trans Amer Neurol Assoc 1964; 89:56-8.

12 Bickford RG, Jacobson JL, Cody D Th. Nature of average evoked potentials to sound and other stimuli in man. Ann NY Acad Sci 1964; 112: 204-18.

13 Meier-Ewert K, Schmidt C, Nordmann G, Hümme U, Dahm J. Averaged muscle responses to repetitive sensory stimuli. In: Desmedt JE, ed. New developments in electromyography and clinical neurophysiology, vol 3. Basel: Karger, 1973: 767-72.

14 Gogan $P$. The startle and orienting reactions in man. A study of their characteristics and habituation. Brain Res 1970; 18:117-35.

15 Rossignol S. Startle responses recorded in the leg of man. Electroencephalogr Clin Neurophysiol 1975; 39:389-97.

16 Shimamura M. Neural mechanisms of the startle reflex in cerebral palsy, with special reference to its relationship with spino-bulbo-spinal reflexes. In: Desmedt JE, ed. New developments in electromyography and clinical neurophysiology, vol 3. Basel: Karger, 1973: 145-58.

17 Marsden CD, Merton PA, Morton HB. Servo action in human voluntary movement. Nature 1972; 238:140-3.

18 Melvin Jones G, Watt DGD, Rossignol S. Eight nerve contributions to the synthesis of locomotor control. In: Stein RB, Pearson KB, Smith RS, Redford JB, eds. Control of Posture and Locomotion. London: Plenum Press, 1973: 579-97.

19 Ertekin C. Studies on the human evoked electrospinogram. I-The origin of the segmental evoked potentials. Acta Neurol Scand 1976; 53:3-20.

20 Ertekin C. Studies on the human evoked electrospinogram. II-The conduction velocity along the dorsal funiculus. Acta Neurol Scand 1976; 53: 21-38.

21 Ertekin C. Evoked electrospinogram in spinal cord and peripheral nerve disorders. Acta Neurol Scand 1978; 57:329-44.

22 Cusick JS, Myklebust J, Larson SJ, Sances A Jr. Spinal evoked potentials in the primate: Neural substrate. J Neurosurg 1978; 49:551-7. 